\title{
REGLA FISCAL, DEUDA PRIVADA Y DERECHOS EN COLOMBIA: LA RECUPERACIÓN DESPUÉS DE LA PANDEMIA*
}

\author{
Diana Alexandra Castañeda Guerrero ${ }^{a}$ \\ Gonzalo Cómbita Mora ${ }^{\mathrm{b}}$ \\ Carolina Castañeda Guerrero ${ }^{\mathrm{C}}$
}

" DOI: https://doi.org/10.18601/01245996.v23n44.10. Recepción: 15-052020, modificación final: 15-05-2020, aceptación: 27-11-2020. Sugerencia de citación: Castañeda G., D. A. (2021). Regla fiscal en Colombia en tiempos de pandemia y post-pandemia. Revista de Economía Institucional, 23(44), 213-231.

a Doctora en Derecho, Universidad Nacional de Colombia, Bogotá, Colombia, [dacastanedag@unal.edu.co], [https://orcid.org/0000-0003-3010-8242].

b Doctor en Ciencias Económicas, profesor en LA Universidad de la Salle y en la Universidad Nacional de Colombia, Bogotá, Colombia, [gcombita@ unisalle.edu.co, gcombitam@unal.edu.co], [https://orcid.org/0000-0001-86725309].

c Doctora en Salud Pública, Universidad Nacional de Colombia, Bogotá, Colombia, [bccastanedag@unal.edu.co], [https://orcid.org/0000-0003-42649476]. 


\section{Regla fiscal, deuda privada y derechos en Colombia: la recuperación después de la pandemia}

Resumen La magnitud de la crisis causada por la pandemia de covid-19 hizo necesario replantear herramientas como la regla fiscal para afrontar situaciones como esta. E1 artículo muestra las bases erradas de la regla fiscal, la relación entre endeudamiento público y privado, y subraya la necesidad de que el Estado deje de actuar como simple espectador y promotor del autocuidado, y asuma la tarea de crear las condiciones necesarias para que la población pueda ejercer la plenitud de sus derechos durante y después de la pandemia.

Palabras clave: regla fiscal, pandemia, COVID-19; JEL: E63, H50

Fiscal rule, private debt and rights in Colombia: the recovery after the pandemic

Abstract Magnitude of the crisis caused by the new coronavirus pandemic generated the need to rethink tools such as the fiscal rule to face these situations. This article raises the wrong bases that sustain the fiscal rule, the relationship between public and private debt, focusing on the need for the State to stop acting as a spectator and promoter only of self-care, so that the necessary conditions are created to ensure the full exercise of the rights of the Colombian population in the post-pandemic scenario.

Keywords: Fiscal Rule, Pandemic, COVID-19; JEL: E63, H50

\section{Regra fiscal, dívida privada e direitos na Colômbia: a recuperação após a pandemia}

Resumo A magnitude da crise provocada pela pandemia covid-19 tornou necessário repensar ferramentas como a regra fiscal para enfrentar situações como esta. $\mathrm{O}$ artigo aponta os equívocos da regra fiscal, da relação entre o endividamento público e privado, e destaca a necessidade de o Estado deixar de atuar como mero espectador e promotor do autocuidado e assumir a tarefa de criar as condições necessárias para a população pode exercer todos os seus direitos durante e após a pandemia.

Palavras-chave: regra fiscal, pandemia, COVID-19; JEL: E63, H50 
Z $\mathrm{n}$ el marco de la crisis económica, social y de derechos humanos Covocada la pandemia de COVID-19 -causada por el SARS$\mathrm{CoV}-2$, que a finales de agosto pasado había contagiado a más de 25,4 millones de personas y ocasionado más de 842.000 muertes en todo el mundo y más de 615.000 casos y 19.600 muertes en $\mathrm{Co}_{-}$ lombia (CRC, 2020) - vuelve a discutirse el papel de los gobiernos en la tarea de encontrar y aportar soluciones a los graves problemas que hoy se deben enfrentar. Las teorías que han fundamentado las decisiones estatales y que se daban por sentadas se deben replantear. Como señaló Steve Keen (2020) el nuevo coronavirus expuso diversas falacias de la economía neoclásica; este artículo se ocupa de las bases de la regla fiscal colombiana.

Hay diferentes reglas, de equilibrio presupuestal, de endeudamiento, de gasto y de ingresos (Cottarelli, 2009). Colombia tiene una regla fiscal de control del gasto público desde el año 2000 (Ley 617/2000) y de equilibrio presupuestal desde 2011 (Acto Legislativo 03/2011Ley 1473/2011), según la cual el gasto estructural no puede superar el ingreso estructural en un monto que exceda la meta anual. De modo que se busca reducir el déficit estructural, que debe ser decreciente: un 2,3\% del PIB en 2014, el 1,8\% en 2018 y el 1\% desde 2022.

La regla colombiana acepta la expansión fiscal cuando la tasa de crecimiento esperada del producto en un año sea al menos dos puntos porcentuales inferior a la tasa de crecimiento económico real de largo plazo; así, permite -durante máximo dos años- una política fiscal contracíclica en caso de emergencias o graves choques macroeconómicos como la crisis de la COVID-19. Por ello, en junio de 2020 el Comité Consultivo para la Regla Fiscal aceptó que el gobierno pudiera realizar gastos extraordinarios hasta por el $20 \%$ de la brecha del producto (CCRF, 2020). Estableció además una excepción a la aplicación de la regla, que funciona como una suspensión, como una válvula de escape a la que se puede recurrir en caso de eventos extraordinarios que amenacen la estabilidad macroeconómica del país.

La regla fiscal $(\mathrm{RF})$ tiene una relación directa con el endeudamiento de los países y surgió para impulsar los ajustes necesarios que hicieran sostenibles las finanzas públicas. Pero, dada la magnitud de la crisis provocada por la COVID-19, la suspensión de la RF y el gasto contracíclico por dos años son insuficientes para afrontarla, $y$ es necesario discutir las bases teóricas y exponer las falencias de esta regla. Lo que se hace en las dos primeras secciones del artículo.

Por otro lado, la austeridad fiscal derivada de la regla crea una presión sobre el endeudamiento privado para recuperar la economía; 
una estrategia de crecimiento fracasada, limitada y peligrosa a nivel mundial. En la tercera sección se estima de manera novedosa el espacio y la historia del crecimiento impulsado por la deuda en Colombia y su impacto en la actual crisis.

Además, el alto desempleo, que en julio de 2020 llegó al 20,2\% (Dane, 2020) afecta de manera directa los derechos de la ciudadanía debido al gran número de hogares que ven reducido su salario -directo, indirecto y diferido-. Un tema que se discute en la cuarta sección.

\section{IDEAS ESENCIALES DE LA REGLA FISCAL}

La idea básica de la RF descansa en un principio moral cristiano, de prudencia y conservadurismo en el manejo de las finanzas públicas. La recomendación bíblica de ahorrar en época de vacas gordas y gastar en época de vacas flacas (Génesis 41:1-36) ilustra la "sensatez", en la cultura occidental, de la RF, una sensatez que trasciende el pensamiento técnico. Onfray (2014) 1lama "principios ateo-cristianos" a este tipo de premisas, pues obedecen a las reglas morales y éticas que construyen el imaginario social.

La teoría económica respalda las tesis de la RF mediante modelos de consumo intertemporal, que suponen que el Estado enfrenta una restricción de recursos para hacer sus gastos, como cualquier individuo; es decir, que debe gastar lo que gana, al menos en el largo plazo. El punto de partida es el trabajo de Fisher $(1907,1999)$ que, con una visión microeconómica basada en el agente representativo, mercados competitivos, precios flexibles, asignación intertemporal de recursos y determinación real de la tasa de interés, muestra que ese individuo maximiza su utilidad endeudándose en un periodo y desahorrando en el periodo posterior para honrar su deuda. Fisher (1930) destaca la bondad de pagar las deudas y subraya frases como "acumular para cuando venga la escasez", que confirman la tesis de Onfray (2014).

Los trabajos de Barro $(1974,1989)$ y Buchanan (1976) aplican las herramientas de Fisher $(1907,1999)$ a un consumidor racional que en sus decisiones intertemporales tiene en cuenta la restricción presupuestal del Estado, un principio conocido como equivalencia ricardiana ${ }^{1}$. Según estos autores, una reducción de impuestos, con un

${ }^{1}$ Ahiakpor (2013) explora el origen del término, que se remonta al capítulo XVII de los Principios de David Ricardo de 1817. Ahiakpor muestra que la versión de Ricardo adaptada por Barro (1974) y Buchanan (1976) desconoce que Ricardo centró su atención en los efectos inmediatos de la sustracción de ahorro a los agentes privados, consumidores y productores, para financiar 
gasto predeterminado del Estado, lleva a que los agentes anticipen perfectamente que luego les cobraran impuestos sobre esta reducción; entonces que mantienen constante el nivel de consumo y ahorran el excedente fruto del alivio tributario. Así, lo único que ocurre es una transferencia intertemporal de recursos equivalente entre privados y Estado, sin afectar la riqueza neta de la sociedad.

Quizá lo más interesante de su conclusión es que la política del Estado tiene un efecto neutral en la demanda agregada, pues no altera las decisiones de consumo de los agentes, y que elimina de tajo el efecto multiplicador keynesiano. No obstante, esta medida "keynesiana" de estabilización se proclama como una medida prudente-ahorrar en época de vacas gordas y desahorrar en época de vacas flacas-, así no sea neutral con respecto al nivel de producción, pues no solo no incentiva la demanda de los agentes privados, sino que el gasto público se limita a ajustarse al nivel de producción predeterminado por los agentes ${ }^{2}$.

El principio de equivalencia ricardiana implica otro efecto neutral: el de la forma de financiamiento del Estado, que es consistente con el teorema de Modigliani y Miller (1958), pues supone que "bajo ciertas condiciones la forma mediante la cual una empresa (Estado) se financia no afecta el costo de capital y no influye en el valor de mercado o en las decisiones de producción o consumo de otros agentes económicos" (Pérez, 2015, 46).

Así, la moral de las finanzas sanas extrapolada a las finanzas públicas, en un sistema de agentes racionales, y que parece ser neutral en materia real y financiera, lleva a pensar que la regla fiscal es un mero instrumento que no afecta los derechos de los ciudadanos; es decir, que debido a su carácter en apariencia instrumental o técnico su cumplimiento es neutro o incuso favorable para los ciudadanos (Zuluaga, Palacios et al., 2011). Esto es puesto en cuestión por la Corte Constitucional, que considera la sostenibilidad fiscal solo como un criterio y no como un principio constitucional (Sentencia C -288 de 2012).

al Estado, es decir, que Ricardo -como buen economista clásico- no pensó en la asignación estática de recursos entre dos periodos sino en los efectos dinámicos de la financiación del Estado en la tasa de acumulación.

2 Como indica la advertencia de Keynes (2006) sobre la limitación del análisis microeconómico de resultados macroeconómicos. El consumo y el gasto del Estado afectan el nivel de producción, y así modifican la restricción presupuestal del agente maximizador. 


\section{EL ESTADO COMO ESPECTADOR DEL AUTOCUIDADO}

E1 gobierno central y sus homólogos locales adoptaron el "autocuidado" como estrategia para promover la salud y contener la enfermedad, el cual requiere un proceso educativo y empoderar a los ciudadanos para que, con información correcta, puedan tomar las mejores decisiones sobre su salud (WHO, 1983, 2009). Sin embargo, para ello se requieren políticas nacionales y locales, así como que el gobierno asigne los recursos necesarios. La falta de políticas públicas no puede trasladar el problema a la autogestión ciudadana, menos aún cuando en la salud de la población intervienen muchos factores que están fuera del control de los individuos.

Algunos de esos factores son de carácter social, cultural, económico y ambiental, como las condiciones de vida, trabajo, vecindario y vivienda; la educación, el saneamiento, las creencias, la nutrición, el acceso a servicios sanitarios, etc. (Dahlgren y Whitehead, 1991; Solar e Irwin, 2010). Todo ellos tienen impacto en la salud y determinan hasta qué punto las personas tienen recursos personales, físicos y sociales para evitar enfermedades y atender su salud, en la medida en que inciden en las elecciones individuales.

Según la lógica del autocuidado, habría que promover el autoempleo para atender el enorme desempleo y el derrumbe de la actividad productiva, no solo durante la pandemia sino como se ha hecho desde las grandes reformas al mercado laboral desde los años setenta (Boyer, 2015; Laval y Dardot, 2013; Setterfield y Cornwall, 2005). Mitchell y Muysken (2008) llaman plena empleabilidad a la situación en la que el trabajo es responsabilidad individual, donde el fracaso en la búsqueda de empleo es resultado de la baja inversión en las habilidades personales. En este enfoque microeconómico el papel del Estado se reduce a garantizar el buen funcionamiento de las fuerzas del mercado de trabajo, es decir, a eliminar toda fricción, como el salario mínimo, la sindicalización o los pagos de seguridad social.

El lavado de manos no es solo una medida de autocuidado durante la pandemia, sino una práctica habitual de los diseñadores de política macroeconómica, que, siguiendo los principios de la macroeconomía clásica y sus nuevas vertientes, están obsesionados por el control de la inflación y el conservadurismo fiscal plasmado en la regla fiscal. Arestis y Sawyer (2004) y Felipe (2012) rechazan del todo medidas como la regla fiscal pues evitan que el Estado siga una política pública de pleno empleo y tenga un papel activo en los procesos de innovación ${ }^{3}$

\footnotetext{
${ }^{3}$ Ver Mazzucato (2019).
} 
y de cambio estructural, el cual es fundamental en la evolución de largo plazo en los países en desarrollo, así como lo ha sido en los países desarrollados. No solo porque la política fiscal incide en las variables reales, a corto y largo plazo, en contra de visiones neoclásicas como la equivalencia ricardiana.

Hodgson (2012) examina los desafíos más profundos a las teorías y prácticas que sustentan la RF. El primero se basa en la noción de dinero como deuda, en la cual el sistema financiero y la moneda son creadas ex nibilo, una concepción ausente en la teoría de la equivalencia ricardiana y sus herederas. E1 dinero se crea en los balances bancarios, cuando en los activos se sienta la cuenta por cobrar y en los pasivos, el depósito a los prestatarios. Este hecho es crucial, pues la financiación de la producción no depende de los depósitos del público originados en la actividad productiva, sino de la demanda de crédito para nuevos proyectos. Así, en un sistema de cero reservas no existe limite a la financiación, y aun en un sistema de reserva fraccionaria, el crédito se podría extender a través del mercado interbancario o préstamos directos del Banco Central (Keynes, 2010). Lo anterior pone en cuestión la equivalencia ricardiana y la forma en que opera la RF, pues el gobierno no necesitaría que los hogares ahorren parte de su ingreso y lo transfieran al gasto público, sino que ambos pueden aumentar su financiación sin entrar en un juego de suma cero en el que uno de ellos debe sacrificarse.

El segundo desafío se deriva de la idea anterior, pues el incremento simultáneo de la demanda de ambos agentes indica que el sistema no está en un equilibrio general, es decir, en el agregado existen excesos de demanda permanentes. Esto obedece a que la función principal del dinero no es el intercambio (M-D-M) ${ }^{4}$, sino la de poner en marcha el sistema capitalista, desde el momento en que los empresarios demandan recursos hasta la realización de la ganancia (D-M-D+) (Graeber, 2014; Keen, 2014; Marx, 2014). En definitiva, se desafía el dogma del equilibrio de la teoría neoclásica y sus efectos sobre la neutralidad monetaria y la dicotomía del sistema. Eso da un golpe rotundo a la RF, uno de cuyos sus cimientos es el concepto de equilibrio. Considerando el desequilibrio permanente de la economía monetaria, Hodgson (2012) plantea que el sistema de crédito nace en desequilibrio, pues en el momento en que hace un crédito, el emisor de la deuda pone en circulación un monto igual de dinero, pero no crea moneda adicional para pagar los intereses, por lo que la posibilidad de honrar el servicio de la deuda en el agregado depende de que otros

${ }^{4} \mathrm{M}$ significa mercancía y D representa el dinero.

Revista de Economía Institucional, vol. 23 , N. ${ }^{\circ} 44$, Primer semestre/202i, Pp. 2 I3-23i ISSN OI 24-5996/E-ISSN 2346-2450 
agentes tomen préstamos en el futuro. Así se desvirtúa el principio de la equivalencia ricardiana que fundamenta la Regla Fiscal, pues supone que el pago de intereses está cubierto de antemano por el valor presente de los impuestos futuros. Además, deja en claro que el pago de la deuda no es una obligación moral sino una imposibilidad fáctica. Y, a su vez, que en una situación de crisis el Estado el que debe tomar créditos para evitar la fragilidad financiera del sistema.

El tercer desafío también se deriva del primero, pues conforme al dinero-deuda, al crearse un crédito nuevo se pone en circulación dinero nuevo, pero cuando se cancelan las deudas el dinero en circulación respaldado por ellas desaparece. Si el gobierno decide cumplir la regla fiscal que lo lleve al balance financiero en el largo plazo, tendría que cancelar toda la deuda, de modo que destruiría el dinero circulante en la economía. Si a esto se suma que los agentes privados deben pagar sus deudas pues no emiten dinero-deuda nuevo, la economía tendría problemas para poner en marcha los procesos de producción, intercambio y distribución, por falta de financiación y de liquidez. Por ello, el déficit es necesario para el buen funcionamiento de la economía.

De aquí se desprende otra idea ligada a una identidad contable básica en macroeconomía: el gasto de alguien es el ingreso de otro; por ello el aumento del déficit público es el que permite ahorrar y acumular activos al sector privado a través de sus ganancias (Mitchell, Wray y Watts, 2019; Wray, 2015). Una política de balance financiero cero del sector público sería perjudicial para la salud financiera de los hogares y de las firmas (López, 2008; López y Assus, 2010).

Un cuarto desafío -que también señalan Mitchell y Muysken (2008), Mitchell et al. (2019) y Wray (2015) - es que los Estados pueden endeudarse en su propia moneda para incentivar la economía en el corto y el largo plazo sin que esto cree problemas financieros, pues a diferencia de los consumidores y empresarios pueden emitir moneda, y es irrelevante hablar de topes de deuda en la búsqueda del pleno empleo, una tesis compatible con las finanzas funcionales (Lerner, 1943). No obstante, para los países en desarrollo sí es un problema endeudarse en moneda extranjera, pues deben exportar para honrar los pagos y así imponen una carga al aparato productivo, es decir, para estos países la restricción es la balanza de pagos (McCombie y Tharnpanich, 2016; Thirlwall, 2003).

El quinto desafío es que la financiación del gasto público no solo es posible, sin restricciones, en la moneda soberana de cada nación sino que se debería financiar con moneda pública, desde el banco central o un sistema financiero público. La ventaja de ese sistema frente a la 
RF es que puede impulsar el crecimiento o la reactivación productiva sin la amenaza de una futura alza de impuestos para pagar a los acreedores privados. Un ejemplo es el New Deal de Roosevelt, que impulsó la recuperación de la economía estadounidense de la crisis de 1929, pero que al ser financiado con recursos del sector financiero privado pronto se tuvo que elevar el recaudo tributaria, lo que frenó la recuperación, que solo llegó con el estallido de la Segunda Guerra Mundial.

Una pregunta obligada para una mente formateada por la teoría neoclásica es el efecto inflacionario de las medidas de financiación pública en moneda propia. En primer lugar, la posibilidad de crear dinero ex nibilo no es exclusiva de la banca pública, el sistema financiero privado también lo crea; el dinero nuevo creado, bien sea por el sistema público o privado, sería igual de inflacionario. En segundo lugar, siempre que el crédito nuevo otorgado permita aumentar la actividad productiva no tiene por qué ser inflacionario. En tercer lugar, en caso de provocar una presión inflacionaria la política tributaria y las operaciones de mercado abierto pueden eliminar los excesos de liquidez de la economía (Wray, 2015).

Como ya se mencionó, quizá la restricción al programa de banca pública para restaurar el pleno empleo en países como Colombia no sea la presión inflacionaria sino la creciente necesidad de importaciones, que desemboca en un creciente déficit comercial, un mayor endeudamiento en moneda extranjera y el colapso de la expansión productiva. Por ello, las políticas de demanda deben estar acompañadas de políticas industriales que alivien la restricción externa. En otras palabras, una política fiscal exitosa necesita subir en la jerarquía de bienes, hacia sectores más complejos, así como se necesita avanzar en la jerarquía de las monedas nacionales, en la soberanía monetaria para usar un término de la teoría monetaria moderna (De Lucci, 2020).

Los elementos anteriores muestran que la RF no es un instrumento neutral ante los derechos de los ciudadanos. Es un mecanismo que los priva de la posibilidad de alcanzar el pleno empleo, de la posibilidad de recuperar la economía en periodos de crisis como la causada por la COVID-19, sin comprometer la viabilidad financiera y la estabilidad macroeconómica a corto y largo plazo. La RF impone a la economía una restricción al crecimiento por la manera de pensar de los diseñadores de política ${ }^{5}$, que se suma a restricciones de los países

${ }^{5}$ Según Castoriadis (2010), la realidad construida es un reflejo del imaginario; en este caso de un modelo prefijado del funcionamiento de la econo- 
en desarrollo como la estabilidad de la balanza de pagos (McCombie y Tharnpanich, 2016).

\section{ENDEUDAMIENTO PÚBLICO Y PRIVADO EN TIEMPOS DE PANDEMIA}

El énfasis en el balance financiero del sector público ha desplazado de facto el problema de endeudamiento al sector privado, mientras que la academia y los medios prestan atención al primero, el segundo se ha desbordado a nivel mundial. Si el Estado no incurre en déficits y busca aumentar sus ingresos somete a los hogares y las firmas a una reducción de su ahorro, y cualquier desequilibrio externo puede afectar directamente el balance privado, lo que es riesgoso en países con alta volatilidad de los ingresos externos, como la caída del precio del petróleo con el estallido de la crisis de la COVID-19. Otros aspectos que refuerzan el endeudamiento privado son la alta propensión a consumir por la emulación (Veblen, 2005), que se ha disparado con la mayor exposición a la publicidad (Cynamon y Fazzari, 2013), y el deterioro de la distribución del ingreso funcional y personal (Lavoie y Stockhammer, 2013; Piketty, 2015).

Keen (2017) propuso el concepto de zona de deuda zoombie ( $z d z$ ) para mostrar los países cuyo motor de crecimiento es la deuda privada. En la $z d z$ los países superan un monto de deuda privada como proporción del PIB es superior al 150\% y un crecimiento promedio de la deuda mayor del 10\% del PIB anual. Keen encontró que en la crisis de 2008 Estados Unidos, Reino Unido, Irlanda, España, Portugal y otros países estaban en la $z d z$, lo que era una seria amenaza para el crecimiento y provocó una gran fragilidad financiera sistémica.

Colombia está lejos de la $z d z$ pues, según el Banco de Pagos Internacional (BPI) en diciembre de 2019 la deuda privada era cercana al 62\% del PIB. No obstante, la gráfica 1 muestra la transición dinámica de la deuda (abcisa) y su tasa de variación (ordenada) durante la depresión de 1999 y las desaceleraciones de 2008 y 2014. Es claro que en 1999 el endeudamiento privado llegó a un nivel histórico que agudizó la crisis. Solo con el boom de bienes básicos de comienzos de siglo mejoró la posición deudora, como se observa en la crisis subprime; luego, la economía fue impulsada con más fuerza por la deuda privada, que aumentó su tasa de crecimiento y su nivel acumulado.

Puesto que la política fiscal ha reducido paulatinamente el déficit como proporción al PIB, con valores de 5,8, 2,8 y 2,3 en los periodos mía. Que algunas situaciones coincidan con posturas ideológicas puede ser mera coincidencia o profecías autocumplidas. 
mencionados, ha ido de la mano con el cumplimiento de la RF y de los principios de austeridad, lo que ha consolidado el papel de la deuda del sector privado como motor de crecimiento junto a la exportación de bienes básicos, en vez de una mayor demanda basada en un mejor nivel de empleo y de remuneración. Esa deuda privada y esas exportaciones pueden impulsar la economía durante un tiempo, pero a medida que la deuda se acumula, luego de superar un umbral, como el de la $z d z$, se torna ineficaz y peligrosa, y provoca estancamiento, como en Japón y otros países ${ }^{6}$.

Gráfica 1

Crédito y deuda privada colombiana como proporción del PIB, 1997-2014

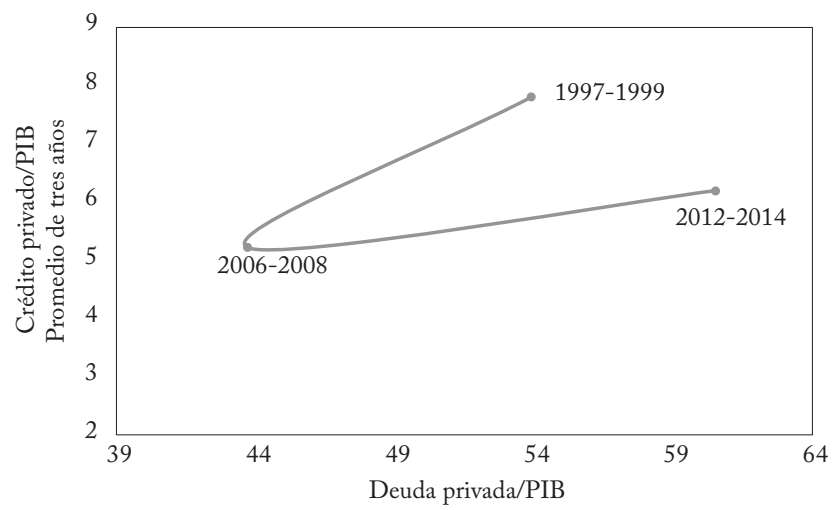

Fuente: BPI, Dane y Banco Mundial; cálculos propios.

La gráfica 2 confirma que en Colombia el crecimiento es impulsado cada vez más por la deuda privada en presencia de políticas de austeridad fiscal, considerando que la demanda agregada es el PIB más la variación del crédito y sus variaciones son las fuerzas detrás del ciclo (Keen, 2014, 2017). Esta gráfica muestra la evolución del PIB real, la demanda agregada real y la brecha entre estas variables como proporción del PIB. En primer lugar, es evidente la capacidad de arrastre de la demanda sobre el PIB real durante los ciclos y la dinámica total del periodo. En segundo lugar, la brecha indica que el impulso de la demanda agregada después de la crisis de 1999 dependió mucho más del crédito. Asimismo, la brecha muestra la estrepitosa caída de la demanda durante la crisis de fin de siglo, que empezó con una desaceleración del crédito

${ }^{6}$ Un ejemplo del problema de $z d z$ es Japón que sufrió una crisis financiera por el estallido de una burbuja financiada con crédito privado (Koo, 2009 y 2011). 
desde 1995, y empeoró hasta alcanzar una caída de 10 por ciento del PIB en el año 2000.

Conociendo el fuerte impacto de la demanda sobre la actividad real, el Estado no debería dudar en intervenir con un mayor gasto financiado con crédito, principalmente en moneda nacional, a través de la banca pública. Así se podría evitar el colapso de la demanda y se generarían ingresos en otros sectores, lo que disminuiría la fragilidad financiera sistémica y haría posible un crecimiento inclusivo, entendido como una expansión real de la actividad económica basada en la creación de empleos bien remunerados (Felipe, 2012).

Gráfica 2

Demanda agregada, PIB y brecha de financiación, 1991-2016

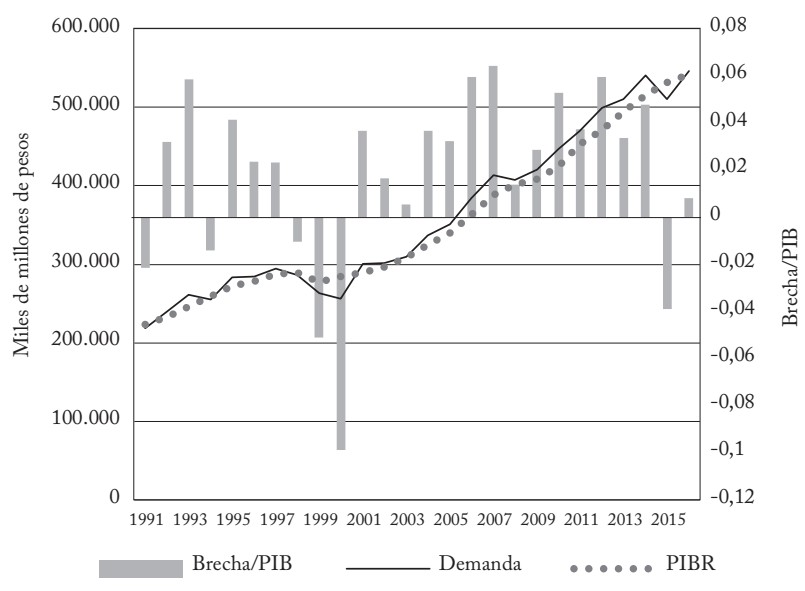

Fuente: Dane, Banco Mundial, cálculos propios.

Keen (2014) elaboró una tabla hipotética para explicar el destino de una economía en la que se desacelera el crédito. El cuadro 1 la reconstruye para el caso colombiano, en el contexto de la COVID $-19^{7}$, con datos de cuentas nacionales y proyecciones oficiales y de entidades reconocidas. Las cifras del cuadro reflejan una posible contracción de la demanda agregada del $42 \%$, cuatro veces mayor que la observada

${ }^{7}$ El cuadro 1 adapta la tabla de Keen con datos colombianos. Crecimiento e inflación son datos de cuentas nacionales y proyecciones oficiales. E1 PIB nominal de 2019 arranca en un valor hipotético de 1.000, que no modifica el análisis luego de aplicar la variación nominal de un año a otro (-4,65\%). La deuda nominal es la cifra del BPI de diciembre de 2019, su crecimiento toma en cuenta el aumento promedio de 2019 y la proyección para 2020. La demanda agregada es la variación de la deuda más el PIB deflactadas a precios de 2019. 
en la crisis de 1999. De nuevo, queda claro el papel estabilizador que puede cumplir una política fiscal activa que absorba los recursos financieros y productivos ociosos, tome créditos nuevos y se financie con moneda pública para restaurar una sana senda de crecimiento en el corto, mediano y largo plazo.

Cuadro 1

Demanda agregada y posible comportamiento macroeconómico, 2019-2020

\begin{tabular}{lcc}
\hline Indicador & 2019 & 2020 \\
\hline Crecimiento real & $3,3 \%$ & $-6,9 \%$ \\
Inflación & $3,8 \%$ & $2,25 \%$ \\
PIB nominal & 1.000 & 953,5 \\
Deuda nominal & 1.618 & 1.715 \\
Tasa de crecimiento de la deuda & $6 \%$ & $0 \%$ \\
Variación de la deuda & 618 & 0 \\
Demanda agregada nominal & 1.618 & 953,5 \\
Demanda agregada real & 1.618 & 933 \\
Cambio en la demanda real & N/A & $-42,4 \%$ \\
\hline
\end{tabular}

Fuente: Dane, FMI; Encuesta de Expectativas Banco de la República, El Tiempo (2020) y FITCH (2020); cálculos propios.

\section{PANDEMIA, REGLA FISCAL, EMPLEO Y DERECHOS FUNDAMENTALES}

La COVID-19 y las cuarentenas masivas impuestas a nivel mundial constituyen la mayor fractura histórica en lo corrido del siglo, y marcará un cambio en todas las sociedades y en el papel que el Estado nacional ha cumplido hasta hoy. En los Estados contemporáneos, la Constitución Política es el texto base que contiene los acuerdos fundamentales de una sociedad, y en el que es cada vez más común incluir la regla fiscal además de los derechos fundamentales,

En Alemania (1985), Singapur (1991), Polonia (1999), Suiza (2003) Francia (2006), Italia (2014) o Dinamarca (2014), la RF ha sido consagrada en la Constitución (IMF, 2016). En los demás países es reglamentada por normas legales nacionales o supranacionales. En Colombia rige la norma de control del gasto público (2000), con una modificación constitucional que incluyó la sostenibilidad fiscal como referente de la acción estatal (2011).

En este escenario institucional llegó la pandemia de la COVID-19, que hizo necesario parar todo el aparato productivo para preservar el derecho a la vida, la salud y la dignidad, con graves efectos sobre los derechos laborales. Hay una relación inescindible entre los derechos laborales y los derechos fundamentales, pues si una persona tiene un 
empleo digno y bien pago puede disfrutar de modo efectivo sus demás derechos - a la salud (a la alimentación, a tratamientos y medicamentos para recuperar la salud), la educación, la vivienda- que inciden en la buena salud y hacen posible el autocuidado.

Si el de desempleo se dispara, como ha sucedido durante la pandemia, y los hogares tienen altas deudas, se afecta en forma manera directa el cumplimiento de los derechos fundamentales. En este punto se debe señalar que los análisis deben tener en cuenta los efectos sobre los salarios directos, indirectos y diferidos ${ }^{8}$.

En caso de desempleo, el salario directo desaparece. En Colombia, el salario diferido por desempleo tiene la forma de un subsidio de desempleo. Debido a la pandemia se creó un Mecanismo de Protección al Cesante que solo ayudó a mantener la afiliación al sistema de seguridad social por tres meses y cobijó a una muy baja proporción de la población desempleada, unas 225.000 personas frente a 794.224 solicitudes (Dinero, 2020). Las otras variantes del salario diferido, como el pago de incapacidades por enfermedades como la COVID-19, solo existen si el trabajador tiene afiliación formal al sistema de seguridad social, y también desaparecen si queda desempleado; además, se diluye la posibilidad de pensionarse y tener una vejez digna.

Por su parte, el salario indirecto - entendido como el acceso efectivo a servicios de salud, servicios públicos, educación, defensa, justicia que hacen posible ejercer los demás derechos fundamentales- se recibe en forma directa por decisiones del gobierno, de modo que es muy sensible al nivel de endeudamiento público y a la regla fiscal.

En la política de empleo también existe una fuerte influencia cristiana, conforme al dictum "el que no trabaja no come" (Tesalonicenses, 3, 6-10), que en el fondo cuestiona moralmente el acceso a los salarios indirectos y diferidos que necesitan las personas para ejercer sus derechos fundamentales cuando no están en condiciones de trabajar, y que desconoce el principio de solidaridad establecido en la Constitución 1991 (Arts. 1, 48, 49, 95, 356, 367).

Las reglas fiscales se han presentado como uno de los instrumentos que sirven para conjurar las crisis (Schaechter, Kinda, Budina y Weber (2012): pero la singularidad de la crisis global ocasionada por la pandemia hace totalmente necesario tomar medidas distintas de las

${ }^{8}$ El salario directo es la remuneración que recibe el trabajador por su trabajo; el salario indirecto corresponde a los servicios que el Estado presta a los trabajadores sin cobrarles un precio o a un precio menor al de mercado, y el salario diferido comprende los derechos que implican recibir un ingreso en situaciones en las que no se puede seguir trabajando: desempleo, enfermedad o vejez (Murillo, 2019). 
que propone la economía ortodoxa (y así se ha hecho en otros países, incluso en Estados Unidos, la cuna de la regla fiscal). Estamos en una recesión profunda, en la que la demanda agregada no alcanza a absorber la capacidad instalada y la oferta potencial de la economía; por ello hay un exceso de trabajo y de instalaciones que permanecen inactivos, el desempleo es muy superior a su nivel habitual (Listokin, 2019) y los derechos de la población son duramente golpeados.

E1 hecho de haber consagrado la sostenibilidad fiscal en la Constitución colombiana ha restado margen de maniobra a los diseñadores de política. En 2022, cuando finalice la fase de gasto contracíclico, la crisis económica y social no se habrá terminado, y el gobierno central siguiente tendrá las manos atadas fiscalmente para garantizar una política de pleno empleo, y tendrá que subir los impuestos y frenar la posible recuperación. Además, cuanta más rigidez constitucional y legal en materia fiscal exista peor será la velocidad de respuesta en épocas de crisis, habrá poco espacio para enfrentar una conmoción similar a la de la COVID-19, o los grandes desastres ambientales provocados por el cambio climático que se prevén.

\section{CONCLUSIONES}

Aunque la regla fiscal y las visiones que la soportan parecen sensatas a primera vista, son técnicamente erróneas, políticamente reaccionarias, y las políticas de austeridad correspondientes han tenido resultados desastrosos en demasiados países. Esos efectos perniciosos se pueden agudizar en los países en desarrollo y en particular en Colombia, cuya población sufría y sigue sufriendo graves penalidades socioeconómicas.

No hay duda de que el autocuidado es un elemento esencial para mantener la salud; pero, sin un entorno propicio para ejercer el autocuidado ocurra, es una obligación vacía y sin posibilidad real de cumplimiento efectivo. Igual ocurre con el empleo, sin las condiciones necesarias para que la fuerza laboral esté ocupada $-y$ en especial en trabajos formales-, la única opción es sobrevivir con falta de derechos. La única salida es que el Estado tenga un papel activo en la coordinación de la sociedad para enfrentar el gran problema de acción colectiva que implica manejar efectivamente la pandemia y sus consecuencias.

Es claro que detrás del discurso técnico de las finanzas sanas de la regla fiscal se esconde un conjunto de ingenuidades teóricas, filosóficas y empíricas. Esos falsos tecnicismos perjudican el interés público $\mathrm{y}$ favorecen intereses privados, en particular la rentabilidad de los 
mercados financieros, que acogen a un gran cliente que renuncia por su cuenta a la financiación pública y decide acrecentar las rentas de la especulación. En la teoría, el principal error de la regla fiscal es tomar como base una explicación en la que están ausentes las realidades observables en una economía monetaria de producción, lo que la hace inútil y estéril. Desde el punto de vista filosófico, la regla muestra el predominio de un imaginario que moldea la realidad hasta que surgen las "anomalías" propias de un sistema regido por el dinero-deuda y de las relaciones de poder, que contradicen los principios neoclásicos que fundan la regla fiscal.

A nivel empírico, se mostró que el consenso sobre la austeridad fiscal desencadenó un problema de balances entre agentes institucionales, en el que el crecimiento es impulsado por el endeudamiento continuo del sector privado, como que se ha hecho evidente en Japón, Estados Unidos y otros países.

Este proceso, que se viene consolidando en Colombia desde el inicio del siglo XXI, se manifiesta en un mayor porcentaje de la demanda financiada con crédito. En este contexto es preocupante que la contracción de la demanda agregada pueda ser cuatro veces mayor que la de 1999 a causa de las políticas de confinamiento y de las medidas casi nulas de sostenimiento del gasto agregado; de modo que si el Estado no interviene con una política fiscal activa, financiada de manera sana-con moneda pública- la debacle no se hará esperar.

En hora de dar cumplimiento al mandato de pleno empleo que establece el artículo 334 de la Constitución Política, el cual fue erróneamente modificado para incluir la sostenibilidad fiscal.

\section{REFERENCIAS}

Ahiakpor, J. C. (2013). The modern Ricardian equivalence theorem: Drawing the wrong conclusions from David Ricardo's analysis. Journal of the History of Economic Thought, 35(1), 77-92.

Arestis, P. y Sawyer, M. (2004). Re-examining monetary and fiscal policy for 21st Century. Cheltenham: Edward Elgar Publishing.

Barro, R. J. (1974). Are government bonds net wealth? Journal of Political Economy, 82(6), 1095-1117.

Barro, R. J. (1989). The Ricardian approach to budget deficits. Journal of Economic Perspectives, 3(2), 37-54.

Biblia (sf). Génesis. [https://www.biblegateway.com/passage/?search= G\%C3\%A9nesis\%2041\&version=RVR1960].

Biblia (sf). Tesalonicenses. [https://www.biblegateway.com/ passage/? se arch $=2 \% 20$ Tesalonicenses $\% 203 \% 3$ A $10-$ 12\&version $=$ RVR1960]. 
Buchanan, J. M. (1976). Barro on the Ricardian equivalence theorem. Journal of Political Economy, 84(2), 337-342.

Boyer, R. (2015). Crecimiento, empleo y equidad: el nuevo papel del Estado. En A. Bárcena y A. Prado (eds.), Neoestructuralismo y corrientes heterodoxas en América Latina y el Caribe s inicios del siglo XXI (pp. 289-313). Santiago de Chile: Comisión Económica para América Latina y el Caribe (CEPAL).

Castoriadis, C. (2010). Institución imaginaria de la sociedad. Barcelona: Tusquest.

Cynamon B. Z. y Fazzari, S. M. (2013). End of the consumer age. En B. Cynamon, S. Fazzari y M. Setterfield (eds.), After the great recession (pp. 129-157). Nueva York: Cambridge University Press.

CCRF. (2020). Comunicado Oficial del Comité Consultivo para la Regla Fiscal, [https://www.minhacienda.gov.co/webcenter/ShowProperty?no deId=\%2FConexion Content\%2FWCC_CLUSTER-128327\%2F\%2Fi $\mathrm{dcPrimaryFile \& revision}=$ latestreleased\#: :text $=]$.

Corte Constitucional (2012). Sentencia C -288.

Cottarelli, C. (2009). Fiscal Rules - Anchoring expectations for sustainable public finances. IMF, [https://www.imf.org/external/np/pp/ eng/2009/121609.pdf].

Dahlgren G. y Whitehead M. (1991). Policies and strategies to promote social equity in health. Background document to WHO. Strategy paper for Europe. Estocolmo: WHO.

Dane. (2020). Gran encuesta integrada de hogares (GEIH). Mercado laboral. [https://www.dane.gov.co/index.php/estadisticas-por-tema/ mercado-laboral/empleo-y-desempleo].

De Lucchi, J. (2020). Teoría monetaria moderna y periferia, [http:// grupolujan-circus.blogspot.com/search/label/de\%20lucchi].

Dinero. (2020). La urgencia de un seguro al desempleo en Colombia, [https://www.dinero.com/pais/articulo/como-funciona-el-subsidio-aldesempleo-en-colombia/293319].

El Tiempo. (2020). Prevén casi nulo crecimiento del crédito y disparo de deudas vencidas, [https://www.eltiempo.com/economia/sectorfinanciero/la-caida-en-el-credito-en-colombia-por-cuenta-del-covid-19-494202].

Felipe, J. (2012). Inclusive growth, full employment and structural change: implications and policies for developing Asia. Londres: Anthem Press.

Fisher, I. (1907). The rate of interest. Nueva York: Martino Publishing.

Fisher, I. (1930). La teoría de la tasa de interés. Madrid: Aosta.

Fisher, I. (1999). La teoría del interés. Madrid: Aosta.

FITCH. (2020). Growth, debt risks underpin Colombia negative outlook. Fitch Ratings, [https://www.fitchratings.com/research/sovereigns/ growth-debt-risks-underpin-colombia-negative-outlook-22-07-2020].

Graeber, D. (2014). En deuda. Una historia alternativa de la economia. Barcelona: Ariel.

Hodgson, E. (2012). La telaraña de deuda. Córdoba: Editorial Almuzara.

IMF. (2016). IMF fiscal rules dataset, [https://www.imf.org/external/ datamapper/fiscalrules/map/map.htm]. 
CRC. (2020). Johns Hopkins Coronavirus Resource Center, [https:// coronavirus.jhu.edu/data/mortality].

Keen, S. (2014). Desenmascarando la economía. México DF.: LAES.

Keen, S. (2017). Can we avoid another financial crisis? Cambridge, UKMalden, Mass.: Polity Press.

Keen, S. (2020). E1 coronavirus expone brutalmente las falacias de la economía neoclásica y la globalización. Revista de Economía Institucional, 22(43), 17-27.

Keynes, J. (2006). Teoría general de la ocupación, el interés y el dinero. México DF: Fondo de Cultura Económica.

Keynes, J. (2010). Tratado sobre el dinero. Madrid: Fundación ICO.

Koo, R. (2009). The holy grail of macroeconomics. New Jersey: John Wiley \& Sons.

Koo, R. (2011). E1 mundo en una recesión de balances. Ensayos Económicos, 63, 7-39.

Laval, C. y Dardot, P. (2013). La nueva razón del mundo. Barcelona: Gedisa.

Lavoie, M. y Stockhammer, E. (2013). Wage led growth: Concept, theories and policies. En E. S. Marc Lavoie (ed.), Wage led growth (pp. 13-39). Nueva York: Palgrave Macmillan.

Lerner, A. P. (1943). Functional finance and federal debt. Social Research, $10(1), 38-51$.

Listokin, Y. (2019). Law and Macroeconomics. Law and Macroeconomics. Cambridge, Mass.: Harvard University Press.

Lopéz, J. (2008). La economía de Michal Kalecki y el capitalismo actual. Ensayos de teoría económica y economía aplicada. México: Fondo de Cultura Económica.

Lopéz, J. y Assous, M. (2010). Michal Kalecki. Londres: Palgrave Macmillan.

Marx, K. (2014). El Capital. Madrid: Akal.

Mazzucato, M. (2019). El estado emprendedor. Barcelona: RBA.

McCombie, J. y Tharnpanich, N. (2016). Balance of payments constrained growth. En An introduction to macroeconomics (pp.282-299). Cheltenham, UK: Edward Elgar.

Mitchell, W. y Muysken, J. (2008). Full employment abandoned: Shitfting sands and policy failures. Cheltenham: Edward Elgar Publishing Limited.

Mitchell, W., Wray, L. R. et al. (2019). Macroeconomics. Londres: Red Globe Press.

Modigliani, F. y Miller, M. (1958). The cost of capital, corporate finance and the theory of investment. American Economic Review, 48(3), 261-297.

Murillo A., J. F. (2019). Crisis, explotación y salarios: análisis marxista del caso español. Análisis Económico, 34(87), 101-124.

Onfray, M. (2014). Tratado de ateología. Barcelona: Anagrama.

Pérez, E. (2015). Una coyuntura propicia para reflexionar sobre los espacios para el debate y el diálogo entre el (neo)estructuralismo y las corrientes heterodoxas. En A. Barcena y A. Prado (eds.), Neoestructuralismo y corrientes heterodoxas en América Latina y el Caribe a inicios del siglo XXI (pp. 145-174). Santiago de Chile: Cepal. 
Piketty. (2015). El capital en el siglo XXI. Santiago de Chile: Fondo de Cultura Económica.

Ricardo, D. (2014). Principios de economía politica y tributación [1817]. Bogotá: Fondo de Cultura Económica.

Schaechter, A., Kinda, T. et al. (2012). Fiscal rules in response to the crisis - A new dataset. IMF WP/12/187.

Setterfield, M. y Cornwall, J. (2005). Una perspectiva neokaldoriana sobre el auge y el declive de la edad de oro. En Setterfied (ed.), La economia del crecimiento dirigido por la demanda (pp. 73-92). Madrid: Akal.

Solar O. e Irwin A. (2010). Conceptual framework for action on the social determinants of health. Social Determinants of Health Discussion Paper 2. Ginebra: WHO.

Thirlwall, A. P. (2003). La naturaleza del crecimiento económico un marco alternativo para comprender el desempeño de las naciones. México DF: Fondo de Cultura Económica.

Veblen, T. (2005). Teoría de la clase ociosa. México DF: Fondo de Cultura Económica.

WHO. (1983). Health education in self-care: Possibilities and limitations, [https://apps.who.int/iris/bitstream/handle/10665/70092/HED_84.1. pdf? sequence $=1$ \&is Allowed $=y]$.

WHO. (2009). Self-care in the context of primary health care: Report of the regional consultation, [https://apps.who.int/iris/handle/10665/206352].

Wray, L. R. (2015). Teoría monetaria moderna: Manual de macroeconomía sobre los sistemas monetarios soberanos. Berlin: Lola Books.

Zuluaga, O., Palacios M. et al. (2011). La sostenibilidad fiscal, un principio para alcanzar los fines del Estado Social de Derecho. Notas Fiscales, 3, 2-16. 